International Journal of Cancer Studies \& Research (IJCR)

1SSN: 2167-9118

\title{
Fractal Entropy: The Return of Unified Theory of Cancer
}

Editorial

McGuinness D

University of Glasgow, College of Medical, Veterinary \& Life Sciences, Institute of Cancer Sciences, Scotland.

\section{*Corresponding Author:}

Dagmara McGuinness

University of Glasgow, College of Medical, Veterinary \& Life Sciences, Institute of Cancer Sciences, Scotland.

Tel: 44 (0)141 2111933

E-mail: Dagmara.McGuinness@glasgow.ac.uk

Received: August 07, 2013

Published: August 27, 2013

Citation: McGuinness D (2013) Fractal Entropy: The Return of Unified Theory of Cancer. Int J Cancer Stud Res. 2(1e), 1-2. doi: http:/ / dx.doi. org/10.19070/2167-9118-130004e

Copyright: McGuinness $\mathbf{D}^{\odot}$ 2013. This is an open-access article distributed under the terms of the Creative Commons Attribution License, which permits unrestricted use, distribution and reproduction in any medium, provided the original author and source are credited.

The concept of a unified theory for cancer is not novel, in fact it was first proposed by Spandidos in 1986[1] where it was postulated that cancer development was an orchestrated series of genetic and epigenetic events which were significantly related to both genetic and environmental factors. In a recent review by Garland[2] another unified theory has emerged and a new model of cancer development has been proposed. In this case, it has been defined as retuning the energy required by a cell to maintain homeostasis, to a state of maximum energy entropy (dissipation) arising from increased cellular dynamics. Garland has postulated that this process can be driven by specific mutations, or other molecular changes, that will re-direct energy flow within a cell to one favouring neoplastic transformation. These changes in energy management within tumour cells have been well established and are generally considered a hallmark of cancer development and progression. In order to survive and escape from homeostatic control, tumour cells need to fine tune their abilities to gain advantage over normal cells, this includes changes in proliferation rate, deregulation of apoptosis and autophagy, changes in cytoskeleton that favour (de-differentiation) increased cell mobility and invasiveness of tumour cells. This theory allows us to look at cell homeostasis as a multidimensional process where proximal or distant events will follow a similar pattern, governed by the redirection of signalling pathways and metabolic processes which release energy i.e., glycolysis; or an event (i.e., mutation, epigenetic change) that unlocks energy production, thus fulfilling the tumour cell's energy requirements. Furthermore, this theory addresses the paradoxical issue of numerous tumour promoting genetic and epigenetic changes leading to the few defining characteristics of cancer. In essence, this bears striking analogy to processes controlling cellular and tissue senescence, as described in the MTR theory of cellular ageing [3].

In fact we can draw an analogy with the natural world when we look at how a neoplastic cell behaves, drawing parallels with sharks and their basic instincts: to eat, reproduce and invade new territories in order to find a better environment for them. Similarly, neoplastic cells are ultimately limited by their environment, ability to proliferate and availability of food/energy and if they don't respond to change quickly enough they will be eliminated. This dynamicity of the neoplastic cell and their ability to by-pass cell security in order to execute their programmes have been studied for decades; and despite the tremendous progress which has been made in the field of cancer biology, we still get surprised by yet another cellular trick.

Garland in his review also postulates that events occurring during cancer induction can be related to fractal networks (Fractal Entropy) that co-ordinate and re-direct entropy to favour 'malignant behaviour' and examines this proposal in an in vitro hematopoietic model of IL-3 dependent cells. The fractal dimension (which describes changes in patterns in relation to the scale by which it is measured), usually exceeds topological dimension and allows us to perceive cells and cellular processes as self-assembled/organised multidimensional and highly dynamic hubs that are interconnected. These are often involved in opposing cellular processes, yet have the capacity for a quick response to environmental stimuli - restoring the balance in a cell to allow it to fulfil its functions. In the case of tumour cells we have this reaches a paradox where the same molecules/pathways, which can execute different functions depending on their temporal and spatial organisation, can both suppress and promote tumourigenesis.

One of the examples used by Garland is the Warburg effect [4], which postulates that energy produced in tumour cells, is linked to a high rate of glycolysis followed by lactic acid fermentation in the cytosol, thus by-passing mitochondria where energy is created via a low rate of glycolysis followed by oxidation of pyruvate in mitochondria [5,6]. Currently, the Warburg effect is generally considered a resultant process, the product of genomic and epigenetic changes, rather than a driving force in tumourigenesis. It has been postulated that in general, tumour cells eliminate mitochondria because they both regulate energy synthesis and are involved in apoptosis, which would ultimately prevent cancer cells from executing their programme. However, the Warburg hypothesis can be related to this new unified theory where the fine-tuning of energy management is critical for tumourigenesis and the genetic/ epigenetic changes are the processes by which it is achieved. It is also very much in keeping with the MTR concept [3].

Of course, when it comes to cancer simplicity is never the answer. In fact other explanations are required to predict how some tumour cells will behave, for example slow growing and dormant resident in our body, or fast progressing malignant cancers. Here Garland combines Chaos theory with Fractal theory to provide 
more complete explanation. This concept is applicable to unstable dynamic systems where a particular outcome is not necessarily defined by the initial event. The hypothesis of fractal entropy fits with the explanation provided by Chaos theory by stipulating that energy management, regardless of how it is achieved, is the central defining tenet of tumourigenesis providing any number of starting points which all lead to the same outcome. Ultimately, the real question is: can we use this unified theory of cancer development in vivo, to advance our understanding, detection and treatment of cancer?

\section{References}

[1]. Spandidos D.A (1986) A unified theory for the development of cancer. Biosci Rep. 6(8):691-708.

[2]. Garland J (2013) Energy management - a critical role in cancer induction? Crit Rev Oncol Hematol. doi: 10.1016/j.critrevonc.2013.04.001. [Epub ahead of print]

[3]. Shiels P.G and Davies R.W (2004) Ageing and death in neurones. In: Davies R.W. and Morris B.J. (eds.) Molecular Biology of the Neuron. Series: Molecular and cellular neurobiology series. Oxford University Press, Oxford, UK, pp. 435-464. ISBN 9780198509981

[4]. Warburg O (1956) On the origin of cancer cells. Science 123 (3191): 309314.

[5]. Sebastián C, Zwaans BM, Silberman DM, Gymrek M, Goren A, Zhong L, Ram O, Truelove J, Guimaraes AR, Toiber D, Cosentino C, Greenson JK, MacDonald AI, McGlynn L, Maxwell F, Edwards J, Giacosa S, Guccione E, Weissleder R, Bernstein BE, Regev A, Shiels PG, Lombard DB, Mostoslavsy (2012) The histone deacetylase SIRT6 is a tumor suppressor that controls cancer metabolism. Cell. 151(6):1185-1199. doi: 10.1016/j. cell.2012.10.047.

[6]. Wang X, Peralta S, Moraes C.T (2013) Mitochondrial alterations during carcinogenesis: a review of metabolic transformation and targets for anticancer treatments. Adv Cancer Res. 119: 127-160. doi: 10.1016/B978-0-12407190-2.00004-6. [Epub ahead of print] 